\title{
A Case of Thoracic Aortic Aneurysm with Hemoptysis as the First Symptom
}

\author{
Han Zhang, Ming Zheng, Jun Bu* \\ Guangzhou Red Cross Hospital, Jinan University, Guangzhou, China \\ Email: *jeanbujun@163.com
}

How to cite this paper: Zhang, H., Zheng, M. and Bu, J. (2020) A Case of Thoracic Aortic Aneurysm with Hemoptysis as the First Symptom. Journal of Biosciences and Medicines, 8, 33-37.

https://doi.org/10.4236/jbm.2020.810004

Received: August 25, 2020

Accepted: October 11, 2020

Published: October 14, 2020

Copyright (อ 2020 by author(s) and Scientific Research Publishing Inc. This work is licensed under the Creative Commons Attribution International License (CC BY 4.0).

http://creativecommons.org/licenses/by/4.0/

\begin{abstract}
This article reports a case of thoracic aortic aneurysm with hemoptysis as the first clinical manifestation, hemoptysis as the first clinical manifestation is very uncommon and to the best of our knowledge, only twenty of such cases have been previously reported. The case report presented here constitutes the longest course of the disease reported in medical literature, and good recovery after thoracic endovascular aortic repair, may help us to increase the understanding and diagnosis of the disease.
\end{abstract}

\section{Keywords}

Case Report, Endovascular Exclusion of Covered Stent, Hemoptysis, Thoracic Aortic Aneurysm

\section{Introduction}

Thoracic aortic aneurysm (TAA) is a common vascular disease that develops slowly but is sudden, fatal and insidious, and its incidence is increasing year by year. Once a thoracic aortic aneurysm ruptures, its disease progresses rapidly. Patients often die quickly due to acute internal hemorrhage or pericardial tamponade. Most patients cannot even survive to complete imaging examinations. This article reports a case of thoracic aortic aneurysm with a small amount of hemoptysis as the first clinical manifestation, to increase the understanding and diagnosis of the disease.

\section{Case Presentation}

A 76-year-old male patient was admitted to the hospital on August 19, 2020 because of "recurrent blood in sputum for 1 month and sudden hemoptysis 200 ml". The patient had blood in sputum with no obvious cause before 1 month, 
and cough after exerting force 1 day ago, followed by bright red blood of about $200 \mathrm{ml}$, accompanied by chest tightness, no chest pain, dizziness, and fatigue; blood test showed hemoglobin $111 \mathrm{~g} / \mathrm{L}$, White blood cell count $10.39 \times 10^{9} / \mathrm{L}$. C-reactive protein: $17.37 \mathrm{mg} / \mathrm{L}$, ECG prompts: sinus rhythm, T wave changes. Blood gas analysis showed that oxygenated hemoglobin was $88.4 \%$; an urgent examination of the pulmonary artery CTA (computed tomography and geography) showed. The descending aorta (about the level of the T7 vertebral body) can be seen with cystic dilation, the size is about $4.6 \times 5.9 \mathrm{~cm}$, both lungs Emphysema, multiple pulmonary bullae formation, enhanced scanning lumen visible ring filling defect shadow, thicker about $1.3 \mathrm{~cm}$. Consider: descending aortic pseudoaneurysm with thrombosis (Figure 1).

Physical examination: T: $36.8^{\circ} \mathrm{C}$. P: 87 beats/minute R: 17 beats/minute BP: $123 / 70 \mathrm{mmHg}$, the percussion sounds of both lungs are over-voiced, and the breath sounds of both lungs are clear and wet rales can be heard. There was no enlargement of the heart circle, HR: $87 \mathrm{bpm}$, the rhythm was neat, no pathological murmur was heard in the auscultation area of each valve, and there was no edema in the limbs. He had a history of hyperuricemia and emphysema for more than 20 years, and a history of trauma to the right eye for more than 10 years. He denied other medical history. Considering that the patient's aneurysm is large and it is difficult to control respiratory activity with other underlying diseases, in order to avoid false aneurysm rupture, intravascular interventional therapy was performed after consultation with family members. Aortic arch angiography showed that the descending aorta showed a breach at about T7 level, false Aneurysm formation. A $34 \mathrm{~mm} \times 200 \mathrm{~mm}$ aortic stent was then placed, and the breach disappeared after another angiography, no contrast agent continued to overflow, blood flow in the left subclavian artery was smooth (Figure 2, Figure 3), the patient's postoperative hemoptysis disappeared and he was discharged 7 days later. Follow-up for 3 weeks, the hemoptysis no longer appear and no other discomfort except for mild chest tightness. An informed consent was obtained from the patient to report the case.

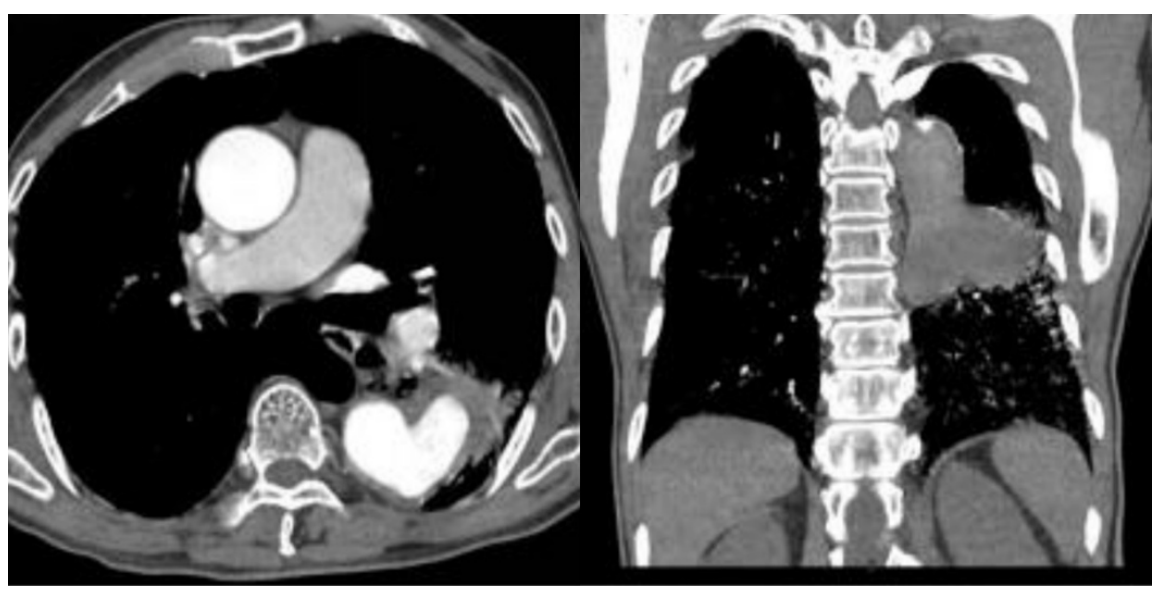

Figure 1. Chest computed tomographyan giography (Before surgery). 


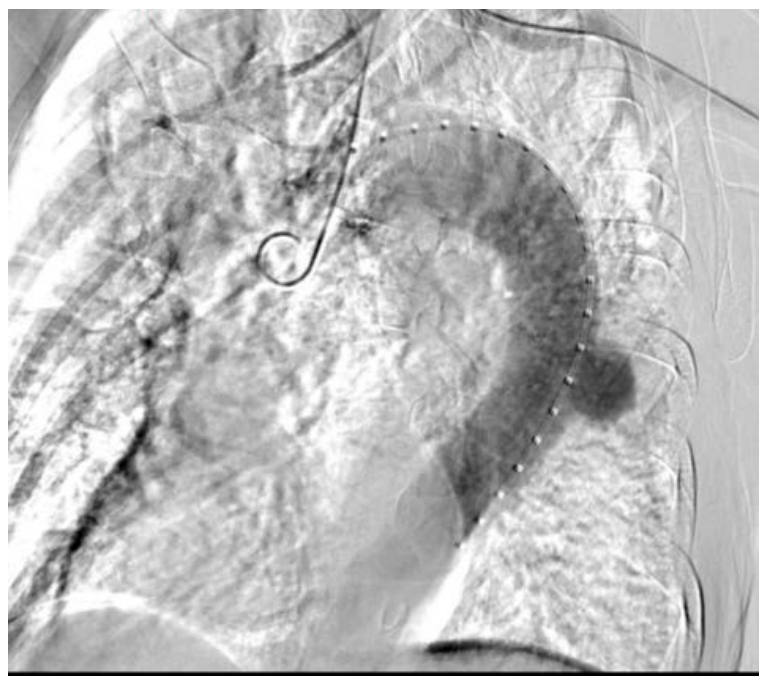

Figure 2. Aortic arch angiography (Before surgery).

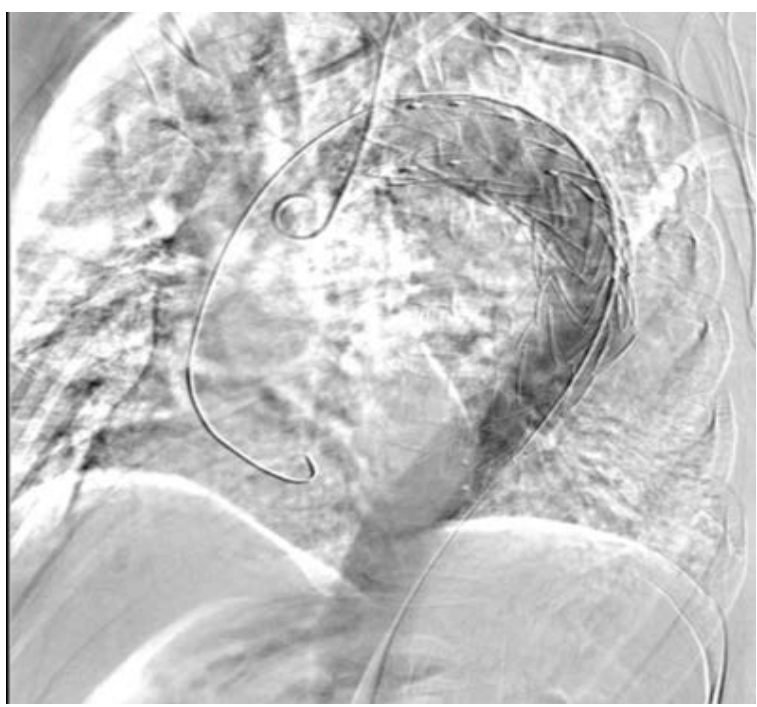

Figure 3. Aortic arch angiography (After surgery).

\section{Discussion}

TAA is a clinically rare disease. Common causes include: aortic atherosclerosis, specific bacterial and viral infections (syphilis, tuberculosis, etc.), cystic necrosis of the vascular media, genetic diseases (Marfan syndrome), and self-Immune diseases (arteritis, Behcet's disease) and trauma, etc [1]. The disease has an insidious onset and no specific symptoms. Most patients with thoracic aortic aneurysms have cough and chest pain. A few patients have difficulty swallowing, breathing, hoarseness, and hemoptysis [2]. Hilary shadows can only be seen on chest imaging examination, and its early symptoms and signs are not obvious. Later, it can cause various symptoms due to tumor enlargement and compression of surrounding tissues [3]. TAA can be divided into true aneurysms and pseudoaneurysms by the integrity of the vessel wall. True aneurysm body is composed of blood vessel wall; pseudoaneurysm is caused by the above-mentioned 
multiple causes, which causes the aorta to rupture and the blood flows out to the surrounding tissues to be wrapped. The most common cause of TAA is atherosclerosis. Refer to the patient's laboratory results and clinical manifestations to rule out genetic diseases, trauma, autoimmune diseases, infections and other factors. Combined with the patient's years of smoking history and CT, multiple calcifications of the aorta and coronary arteries can be seen, and the location of the pseudoaneurysm breach is in calcification severe areas, atherosclerosis is still considered as the cause of the disease in this patient.

Thoracic aortic aneurysms with a small amount of hemoptysis as the initial symptom are rare in clinical practice, and only case reports can be seen in domestic and foreign literature. Symptoms of hemoptysis may include abnormal communication between bronchial segments and enlarged thoracic aortic aneurysm. It is a rare cause of hemoptysis. It is also rare in thoracic aortic aneurysms. Patients with this disease have a history of emphysema for many years. Excluding early hemoptysis is caused by chronic bronchitis, which leads to increased bronchial and capillary permeability, and rupture of small blood vessels under the mucosa, which also brings certain difficulties to early diagnosis. At the same time, we don't think that a small amount of hemoptysis is caused by a pseudoaneurysm breaking into the bronchus. Due to the lack of resistance in the bronchus, the occurrence of hemoptysis at this time is often fatal [4]. Aggregate multiple research reports [5] [6]. After the patient has a small amount of hemoptysis, symptomatic sedation, blood pressure reduction, heart rate control and application of hemostatic drugs can temporarily control the symptoms. However, if the cause is not resolved, the thoracic aortic aneurysm has symptoms of massive hemoptysis and the mortality rate without surgical or interventional treatment Is $100 \%$. The patient has a long course, this may be the reason why he was lucky enough to be treated, but we should have a view: hemoptysis may be due to TAA.

\section{Conclusion}

Due to the extremely high mortality rate of the disease, the 5-year survival period of untreated patients is only $10 \%$. Therefore, for patients suspected of having thoracic aortic aneurysm accompanied by hemoptysis, imaging examinations such as CTA should be perfected to avoid misdiagnosis and miss diagnosis while confirming the diagnosis. For patients with surgical indications, it is recommended to actively perform surgical treatments such as thoracic endovascular aortic repair (TEVAR).

\section{Conflicts of Interest}

The authors declare no conflicts of interest regarding the publication of this paper.

\section{References}

[1] Erbel, R., Aboyans, V., Boileau, C., et al. (2014) 2014 ESC Guidelines on the Diag- 
nosis and Treatment of Aortic Diseases: Document Covering Acute and Chronic Aortic Diseases of the Thoracic and Abdominal Aorta of the Adult. The Task Force for the Diagnosis and Treatment of Aortic Diseases of the European Society of Cardiology (ESC). European Heart Journal, 35, 2873-2926.

https://doi.org/10.1093/eurheartj/ehu281

[2] Fok, M., Bashir, M., Goodson, N., et al. (2017) Thoracic Aortic Aneurysms in Behcet's Disease. Rheumatology (Oxford), 56, 501-502.

[3] Ramanath, V.S., Oh, J.K., Sundt, T.R., et al. (2009) Acute Aortic Syndromes and Thoracic Aortic Aneurysm. Mayo Clinic Proceedings, 84, 465-481. https://doi.org/10.1016/S0025-6196(11)60566-1

[4] Chen, J.H., Ishikawa, T., Michiue, T., et al. (2011) An Autopsy Case of Unexpected Sudden Death due to Rupture of a Thoracic Aortic Aneurysm into the Left Lung. Legal Medicine ( Tokyo), 13, 201-204. https://doi.org/10.1016/j.legalmed.2011.02.001

[5] Ioannidis, O., Trellopoulos, G., Tamouridis, G., et al. (2012) A Single-Centre Experience of the Treatment of Ruptured Abdominal Aortic Aneurysms: Clinical and Anatomic Characteristics of Open versus Endovascular Repair. International Angiology, 31, 386-392.

[6] Chen, X.H., Sun, X.Y. and Cao, D.B. (2012) Three Cases of Hemoptysis Caused by Thoracic Aorticpseudoaneurysm Breaking into the Lung. Chinese Journal of Clinicians (Electronic Edition), 6, 6183-6185. 